\title{
Uso del Web of Science para el estudio de disciplinas mi- noritarias en Humanidades: el caso del folclore
}

\author{
Jordi Ardanuy
}

Artículo recibido:

2 de febrero de 2015

Artículo aceptado:

15 de abril de 2016

\section{Resumen}

El objetivo de este artículo es mostrar la utilidad del Web of Science para obtener información sobre la producción e impacto del Folclore, área minoritaria dentro de las Humanidades. Hasta donde conocemos no existe ningún análisis bibliométrico sobre dicha disciplina. El presente artículo pretende también mostrar las principales características del área emanadas del propio estudio. Para la recuperación de registros se ha utilizado la categoría de WoS "Folklore", mientras que el impacto se ha obtenido principalmente a partir de los informes de citas que ofrece la misma base de datos. Los resultados muestran un gran volumen de reseñas de libros. La colaboración entre autores es todavía

* Universidad de Barcelona Catalonia, España. jordi_ardanuy@ub.es 
poco frecuente, mientras que sí es usual la publicación en lenguas diferentes del inglés. El uso de WoS permite identificar otra información relevante sobre el área, pese a las limitaciones que ofrece para un estudio global del comportamiento bibliométrico de la disciplina.

Palabras clave: Bibliometría; Folclore; Humanidades; Web of Science; Scopus.

Using the Web of Science for the study of lesser disciplines in Humanities: the case of folklore

Jordy Ardanuy

\section{Abstract}

This paper endeavors to show the usefulness of Web of Science (WoS) in obtaining information about production and impact of Folklore within the larger framework of the Humanities. As yet, there has been no general bibliometric analysis of the discipline. The main features of the field, emanating from the study itself, are described. Using an advanced search protocol, data were retrieved from the Web of Science for the category "Folklore". The distribution of citations of each paper was obtained using the Citation Report tool. Results show the great importance of book reviews. Collaborative authorship is still unusual, whereas authors frequently publish in languages other than English. Despite its limitations in with regard to performing a comprehensive bibliometric study of the behavior of a discipline, the use of WoS data allows the identification of other relevant information from the field of folklore.

Keywords: Bibliometrics; Folklore; Humanities; Web of Science; Scopus.

\section{INTRODUCCIÓN}


producción significativa en lenguas diferentes al inglés (Hicks, 2004; Moed, 2005; Nederhof, 2006; Ardanuy, Urbano y Quintana, 2011; Aghaei Chadegani et al., 2013). Para algunas disciplinas, herramientas como Journal Citation Report (JCR) o SCImago Journal \& Country Rank (SJR) ni siquiera existen. La consecuencia de todo ello es que el número de estudios en humanidades es bastante limitado si se comparan con las ciencias experimentales o incluso con las ciencias sociales (Stone, 1982; Van Impe y Rousseau, 2006). Consecuentemente, Ardanuy (2013a) encontró que solamente el $22.2 \%$ de los análisis de citas en humanidades estaba basado en índices de citas, lo que implica que un $77.8 \%$ procedía de una extracción ad boc de referencias, pese a que un $62.3 \%$ de los trabajos versaba sobre artículos de revista.

Ciñéndonos a la disciplina del folclore, hasta tiempos recientes le ha sido difícil distinguirse nítidamente de otras áreas cercanas tales como la antropología cultural o la historia, con la consecuente falta de identidad académica y presencia duradera en los planes de estudio de la educación superior (Dundes, 2005; Widdowson, 2010). Esto no ha facilitado tampoco la proliferación de análisis bibliométricos sobre este campo de conocimiento. En la revisión bibliográfica aparecen algunos artículos centrados en el estudio de títulos de revista específicos. Así, Calvo (2002), en su trabajo sobre la Revista de Dialectología y Tradiciones Populares, analizó su contenido entre 1944 y 1996 ofreciendo algunos datos estadísticos en un anexo. Cuatro años más tarde, Chang (2006) llevó a cabo un análisis bibliométrico de la revista china Folklore Studies. Yalçin (2010) analizó la revista turca Millî Folklor entre los años 2007 a 2009 a partir de la base de datos WoS. Un artículo de Ardanuy (2013b) mostró el impacto del uso de fuentes primarias en los análisis de citas en el área del folclore usando datos de la revista catalana L'Upir. Todos estos trabajos fueron publicados en las revistas analizadas y en sus respectivos idiomas.

Por su parte, Rudy (2002) estudió la contribución de Verbal Art as Performance, del autor Richard Bauman, en los estudios folclóricos y otros campos académicos. Para ello realizó un análisis de citas en el Social Sciences Citation Index (SSCI) y en el Arts and Humanities Citation Index (A\&HCI). Thukakwenzeka (2003) identificó y analizó la bibliografía sobre los conocimientos indígenas en Sudáfrica (1991-2002) disponible en SABINET. Más recientemente, Zheng (2009) realizó un análisis bibliométrico del patrimonio cultural intangible de China (2004-2008). Su artículo, basado en la base de datos de revistas académicas chinas (CAJD, por sus iniciales en inglés), encontró un alto grado de material multidisciplinar relacionado con el folclore. Sin embargo, ninguno de estos trabajos ofrece una panorámica general sobre el área.

El objetivo principal del presente artículo es mostrar cómo el Web of Science puede aportar información sobre la producción e impacto en una 
disciplina como el folclore, además de ser una primera aproximación bibliométrica a la misma. En agosto de 2011, Thomson Reuters lanzó la versión 5 de Web of Knowledge (WoK), actualmente Web of Science (WoS). Entre otros cambios y adiciones, las 222 antiguas categorías temáticas ISI de la versión 4 del WoK fueron retituladas y se extendieron a 225 términos de categoría WoS (Leydesdorff, Carley y Rafols, 2013), y más tarde a 251. Estas categorías son asignadas por el personal de WoS de acuerdo a varios criterios tales como el título de la revista, sus patrones de citación, etc. (Leydesdorff y Rafols, 2009). En marzo de 2012, la versión 5.6 incorporó una búsqueda por categorías WoS en la búsqueda avanzada. Esta función es la utilizada en el presente estudio con el fin de recuperar los registros de la categoría "folclore" utilizada en el A\&HCI. De acuerdo con su nota de aplicación de Thomson Reuters, esta categoría se utiliza para identificar documentos sobre tradiciones, traje folclórico, narración de cuentos, comida, música tradicional, juegos infantiles, bodas, entierros y otras costumbres. Puesto que A\&HCI no dispone hasta ahora de un JCR, WoS no utiliza esta categoría con la finalidad de calcular el impacto de tales revistas.

\section{Metodología}

El conjunto de registros bibliográficos utilizados en este trabajo se obtuvo a partir de la versión en línea de Web of Science en octubre de 2013, todavía Web of Knowledge en ese momento. Está constituido por los documentos correspondientes a la categoría "folclore" de WoS publicados hasta el año 2012 inclusive, así como las citas que cada uno había recibido hasta el final de ese año. Para ello se utilizó una ecuación bien simple en la búsqueda avanzada: documentos que cumplieran que "WC=(Folklore)" y se hubieran publicado hasta 2012 inclusive. Se utilizó la función Informe de citas (Citation report) para obtener los datos sobre las citas recibidas por cada uno de los documentos. Puesto que esta herramienta incluía las citas del entonces año en curso en ese momento (2013), se excluyeron manualmente con posterioridad. Esta herramienta también ha sido utilizada para medir el índice $b$ de cada revista, seleccionando en cada caso los artículos correspondientes y cuidando la precisión de los datos de manera manual.

Los resultados de la búsqueda fueron exportados a una base de datos relacional y se filtraron de forma manual con el fin de evitar duplicados y corregir errores. En muchos casos, especialmente con los documentos más antiguos, WoS identifica a los autores con un apellido y unas iniciales, además 
de generar confusiones al tratar el primer apellido de algunos autores como parte del nombre de pila. En la medida de lo posible se ha procurado identificar a los autores a partir de la información contenida en los artículos originales o en bases de datos bibliográficas alternativas donde estuvieran indexados. Cuando este procedimiento no ha permitido una identificación suficiente, se ha recurrido a la información institucional de las universidades e institutos de investigación a los que estuvieran afiliados, a notas biográficas y obituarios, así como a las citas y comentarios de trabajos posteriores en los que se hacía referencia al trabajo aludido.

Puesto que el A\&HCI no dispone de un JCR, se utilizaron los datos del Scimago Journal Rank para calcular factores de impacto de las revistas categorizadas como "folclore" en WoS. La selección de títulos fue manual, al no existir en Scopus una categoría equivalente a la de WoS.

\section{Resultados Y DisCusióN}

\section{Tipos de documentos, idiomas y áreas disciplinares}

El volumen total de registros obtenidos en WoS es de 33656 (6.7 \% corresponde a acceso abierto), de los cuales el 57 \% son reseñas de libros, alrededor del $28.9 \%$ está formado por artículos de revistas, y el $0.9 \%$ corresponde a actas de congreso (proceedings papers). El resto corresponde a otros tipos de documentos como materiales editoriales, notas, opiniones, artículos biográficos, etc. (Tabla 1). La naturaleza prolífica de las reseñas de libros es superior al porcentaje total en el A\&HCI. Según Zuccala y van Leeuwen (2011), en el periodo 1981-2009 este porcentaje fue aproximadamente del 45 \%. En el mismo periodo de tiempo, el presente estudio encontró que alcanzaba el $57 \%$.

Como se sabe, las reseñas consisten en escritos breves que analizan una o más obras de interés científico, abordando la relevancia para la investigación de un tema en determinado momento. Suelen constar de un solo epígrafe y se presentan siguiendo una estructura argumentativa. En el caso que nos ocupa, las reseñas no cubren únicamente trabajos académicos o reediciones o traducciones de textos antiguos y antologías de relatos, sino que abarcan material divulgativo sobre leyendas, guías turísticas con material sobre folclore e incluso publicaciones con contenido de tipo oculto o paranormal. 
Tabla 1. Tipos de documentos

\begin{tabular}{|c|c|c|c|c|c|}
\hline \multirow[b]{2}{*}{ Tipo de documento } & \multicolumn{3}{|c|}{ Volumen de documentos } & \multicolumn{2}{|c|}{ Citas } \\
\hline & $\begin{array}{c}\mathrm{N}^{0} \text { de } \\
\text { documentos }\end{array}$ & $\begin{array}{c}\mathrm{N}^{0} \text { de } \\
\text { documentos }\end{array}$ & $\begin{array}{l}\text { Mediana citas } \\
\text { recibidas }\end{array}$ & $\begin{array}{l}\text { Media Citas } \\
\text { recibidas }\end{array}$ & $\begin{array}{c}\% \text { de } \\
\text { documentos } \\
\text { citados }\end{array}$ \\
\hline Reseña de libro & 19197 & $57.0 \%$ & 0 & 0.02 & $6.9 \%$ \\
\hline Artículo & 9726 & $28.9 \%$ & 0 & 1.45 & $38.3 \%$ \\
\hline Material editorial & 947 & $2.8 \%$ & 0 & 0.29 & $21.6 \%$ \\
\hline Nota & 693 & $2.1 \%$ & 1 & 0.56 & $50.8 \%$ \\
\hline Record review & 420 & $1.2 \%$ & 0 & 0.03 & $3.3 \%$ \\
\hline $\begin{array}{l}\text { Ítem sobre un } \\
\text { individuo }\end{array}$ & 379 & $1.1 \%$ & 0 & 0.09 & $9.2 \%$ \\
\hline Ítem biográfico & 348 & $1.0 \%$ & 0 & 0.06 & $5.7 \%$ \\
\hline Reseña & 327 & $1.0 \%$ & 0 & 1.08 & $79.2 \%$ \\
\hline $\begin{array}{l}\text { Reseña } \\
\text { cinematográfica }\end{array}$ & 321 & $1.0 \%$ & 0 & 0.08 & $8.0 \%$ \\
\hline $\begin{array}{l}\text { Actas (Proceedings } \\
\text { paper) }\end{array}$ & 314 & $0.9 \%$ & 0 & 0.68 & $23.6 \%$ \\
\hline Cartas & 299 & $0.9 \%$ & 0 & 0.10 & $9.4 \%$ \\
\hline $\begin{array}{l}\text { Otros (poemas, } \\
\text { noticias, ficción, } \\
\text { otras reseñas, ...) }\end{array}$ & 685 & $2.0 \%$ & 0 & 0.18 & $9.3 \%$ \\
\hline Total & 33656 & $100.0 \%$ & 0 & 0.47 & $15.4 \%$ \\
\hline
\end{tabular}

Si consideramos el total de documentos, solamente un $15.4 \%$ ha obtenido al menos una cita. Desglosado, el $38.3 \%$ de los artículos han sido citados y en cambio solamente el $6.9 \%$ de las reseñas. Situaciones análogas con gran presencia de reseñas de libros y, por contra, una ratio baja de ítems citados ya había sido observada en el caso de la literatura y de la historia (Zuccala y van Leeuwen, 2011).

El volumen total de citas asciende a 256924 . No se han identificado bibliográficamente de manera sistemática, pero una parte considerable corresponde a monografías y libros que constituyen fuentes analizadas (fuentes primarias). Esta observación es totalmente consistente con estudios anteriores en humanidades (Ardanuy, Urbano y Quintana, 2009; Engels, Ossenblok y Spruyt, 2012; Osca-Lluch, Veyrat y Morales, 2013). En el caso específico del folclore, Yalçin (2010) encontró en su estudio sobre Millî Folklor que el 
$70.5 \%$ de todas las citas eran de libros y Ardanuy (2013b) halló casi un 50 \% en L'Upir. Por otro lado, las citas a los artículos de las revistas de folclore indexadas en WoS suman un total de 15855.

WoS dispone de artículos indexados con la categoría de folclore que se remontan hasta 1956, pero no aparece un número significativo de títulos de revista hasta 1975, coincidiendo con la aparición del A\&HCI (Figura 1). Antes de 1975, la revista Journal of American Folklore es la única fuente documental indexada, excepto entre 1966 y 1968, en que también lo está Acta etbnographica Academiae Scientiarum Hungaricae. Naturalmente, el volumen total de registros por año está estrechamente relacionado con esta situación, lo que puede verse en la Figura 1. Es conveniente señalar que el máximo de documentos anuales se alcanza en el año 1980.

Figura 1. Número de títulos de publicaciones, número total de documentos y número de artículos

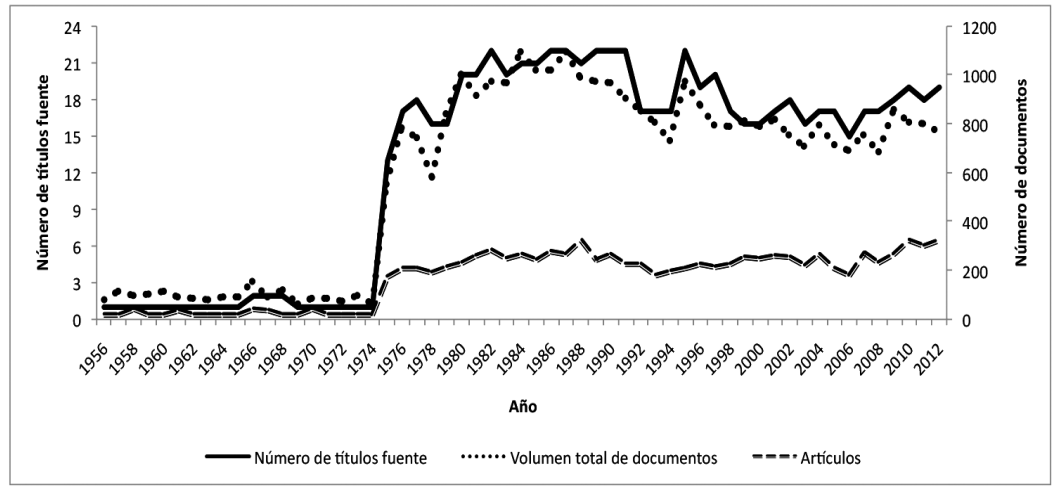

Si nos centramos en las fuentes, aparecen un total de 29 títulos de revista, dos de los cuales han sufrido un cambio significativo de nombre. Diez de estas revistas $(34.5 \%$ ) tienen su sede en EUA, cuatro en el Reino Unido, tres en Alemania y 12 en diferentes países (Tabla 2). En 15 casos (51.7 \%) publican sólo en inglés, uno en francés y uno en alemán. En contraste, 13 revistas $(44.8 \%$ ) han publicado artículos en dos o más idiomas. Por otra parte, también se han indexado 21 títulos de actas de congresos.

Del total de documentos en revistas, el $29.3 \%$ fueron publicados en revistas estadounidenses (33.4\% si sólo se consideran los artículos propiamente); $27.1 \%$ en revistas alemanas ( $13.4 \%$ de artículos); $11 \%$ en revistas de Reino Unido (13.1\%); $7.3 \%$ de Austria (5.9\%); $6.9 \%$ de Suiza (4.1 \%); 5 \% de Japón (4.9 \%); 4.2 \% de Bélgica (5.4\%) y $2.9 \%$ de España (6 \%). 
Tabla 2. Revistas de Folclore en WoS

\begin{tabular}{|c|c|c|c|c|c|c|}
\hline Título & $\begin{array}{l}\text { Organización } \\
\text { responsable }\end{array}$ & $\begin{array}{l}\text { Idioma } \\
\text { principal }\end{array}$ & $\begin{array}{l}\text { Otros } \\
\text { idiomas }\end{array}$ & $\begin{array}{c}\% \text { de } \\
\text { documentos } \\
\text { del total }\end{array}$ & $\begin{array}{c}\% \text { de } \\
\text { artículos } \\
\text { del total }\end{array}$ & $\begin{array}{c}\text { Principales contri- } \\
\text { buidores (países } 0 \\
\text { áreas regionales } \\
\text { con más de un } 5 \% \\
\text { de artículos en la } \\
\text { revista) }\end{array}$ \\
\hline $\begin{array}{l}\text { Acta ehnographica } \\
\text { Academiae Scientia- } \\
\text { rum Hungaricae }\end{array}$ & $\begin{array}{l}\text { Akadémiai Kiadó } \\
\text { (Academia Húngara } \\
\text { de Ciencias) }\end{array}$ & Inglés & $\begin{array}{l}\text { Francés } \\
\text { Alemán }\end{array}$ & $1.6 \%$ & $1.5 \%$ & Hungría (72.0 \%) \\
\hline $\begin{array}{l}\text { Asian Ethnology } \\
\text { Continued by: } \\
\text { Asian Folklore } \\
\text { Studies }\end{array}$ & $\begin{array}{l}\text { Nanzan University } \\
\text { (Japón) }\end{array}$ & Inglés & & $4.9 \%$ & $4.9 \%$ & $\begin{array}{l}\text { EUA (40.6 \%); Países } \\
\text { asiáticos ( } 27.2 \%) \text {, } \\
\text { incluyendo Japón } \\
(8.3 \%) \text { y China } \\
(5.8 \%) ; \\
\text { RU }(5.4 \%)\end{array}$ \\
\hline Fabula & $\begin{array}{l}\text { International Society } \\
\text { for Folk Narrative } \\
\text { Research (Alemania) }\end{array}$ & Inglés & $\begin{array}{l}\text { Francés } \\
\text { Alemán }\end{array}$ & $9.0 \%$ & $5.9 \%$ & $\begin{array}{l}\text { Alemania (28.2\%); } \\
\text { EUA (21.7\%); } \\
\text { Israel }(5.5 \%) ; \\
\text { Suiza }(5.3 \%)\end{array}$ \\
\hline FFCommunications & $\begin{array}{l}\text { Folklore Fellows, } \\
\text { Academia Scientia- } \\
\text { rum Fennica (Aca- } \\
\text { demia Finlandesa de } \\
\text { Ciencias } \\
\text { y Letras) }\end{array}$ & Inglés & $\begin{array}{l}\text { Francés } \\
\text { Alemán } \\
\text { Español } \\
\text { Holandés }\end{array}$ & $0.6 \%$ & $1.4 \%$ & $\begin{array}{l}\text { EUA (25.8 \%); } \\
\text { Finlandia (23.7\%); } \\
\text { RU (18.3\%); } \\
\text { Suiza (12,9\%); } \\
\text { Noruega (5.4 \%) }\end{array}$ \\
\hline $\begin{array}{l}\text { Folk Life, Journal of } \\
\text { Ethnological Studies }\end{array}$ & $\begin{array}{l}\text { Society for Folk Life } \\
\text { Studies (Gales, RU) }\end{array}$ & Inglés & & $0.2 \%$ & $2.8 \%$ & $\begin{array}{l}\text { RU (76.7 \%); } \\
\text { Irlanda (6.7\%) }\end{array}$ \\
\hline Folk Music Journal & $\begin{array}{l}\text { English Folk Dance } \\
\text { and Song Society }\end{array}$ & Inglés & & $3.5 \%$ & $1.5 \%$ & $\begin{array}{l}\text { RU (75.8 \%); } \\
\text { EUA (12.1\%) }\end{array}$ \\
\hline Folklore & $\begin{array}{l}\text { The Folklore Society } \\
\text { (Inglaterra, RU) }\end{array}$ & Inglés & & $5.7 \%$ & $6.3 \%$ & $\begin{array}{l}\mathrm{RU}(44.6 \%) \\
\text { EUA }(26.7 \%)\end{array}$ \\
\hline Folklore Americano & $\begin{array}{l}\text { Instituto Panameri- } \\
\text { cano de Geografía e } \\
\text { Historia (México) }\end{array}$ & Español & $\begin{array}{l}\text { Portugués } \\
\text { Inglés }\end{array}$ & $1.0 \%$ & $2.3 \%$ & $\begin{array}{l}\text { América Latina } \\
\text { (78.3\%), incluyendo } \\
\text { Guatemala (18.3\%), } \\
\text { Argentina (15.0\%), } \\
\text { Costa Rica }(6.1 \%), \\
\text { (Brasil (5.6\%); } \\
\text { Chile (5.6\%); } \\
\text { EUA (16.1\%) }\end{array}$ \\
\hline $\begin{array}{l}\text { Folklore: Electronic } \\
\text { Journal of Folklore }\end{array}$ & $\begin{array}{l}\text { Eesti Keele Instituut } \\
\text { (Instituto de Filolo- } \\
\text { gía de Estonia) }\end{array}$ & Inglés & & $0.6 \%$ & $1.2 \%$ & $\begin{array}{l}\text { Estonia (34.9\%); } \\
\text { Rusia (11.9\%); } \\
\text { Finlandia (9.2\%): } \\
\text { Grecia (6.4\%) }\end{array}$ \\
\hline $\begin{array}{l}\text { International Journal } \\
\text { of Oral History }\end{array}$ & $\begin{array}{l}\text { Alan M, Meckler } \\
\text { (EUA) }\end{array}$ & Inglés & & $0.9 \%$ & $1.1 \%$ & $\operatorname{EUA}(70.4 \%)$ \\
\hline
\end{tabular}


USO DEL WEB OF SCIENCE PARA EL ESTUDIO DE DISCIPLINAS MINORITARIAS...

\begin{tabular}{|c|c|c|c|c|c|c|}
\hline $\begin{array}{l}\text { Jahrbuch fur Volks- } \\
\text { liedforschung } \\
\text { Continued by: } \\
\text { Lied und Populare } \\
\text { Kultur Song and } \\
\text { Popular Culture }\end{array}$ & $\begin{array}{l}\text { Deutsche Volkslie- } \\
\text { darchiv (Archivo de } \\
\text { música popular de } \\
\text { Alemania) }\end{array}$ & Alemán & $\begin{array}{l}\text { Inglés } \\
\text { Francés }\end{array}$ & $7.2 \%$ & $3.0 \%$ & $\begin{array}{l}\text { Alemania }(60.1 \%) ; \\
\text { EUA }(13.1 \%) ; \\
\text { Austria }(6.5 \%)\end{array}$ \\
\hline $\begin{array}{l}\text { Journal of American } \\
\text { Folklore }\end{array}$ & $\begin{array}{l}\text { American Folklore } \\
\text { Society (EUA) }\end{array}$ & Inglés & & $14.7 \%$ & $12.4 \%$ & $\begin{array}{l}\text { EUA (88.5 \%); } \\
\text { Canadá (5.0\%) }\end{array}$ \\
\hline $\begin{array}{l}\text { Journal of the } \\
\text { Folklore Institute, } \\
\text { Continued by: } \\
\text { Journal of Folklore } \\
\text { Research }\end{array}$ & $\begin{array}{l}\text { Folklore Institute at } \\
\text { Indiana University }\end{array}$ & Inglés & & $2.1 \%$ & $5.1 \%$ & EUA (69.6\%) \\
\hline $\begin{array}{l}\text { Journal of Latin } \\
\text { American Lore }\end{array}$ & $\begin{array}{l}\text { Latin American } \\
\text { Institute (EUA) }\end{array}$ & Inglés & Español & $0.3 \%$ & $1.0 \%$ & $\begin{array}{l}\text { EUA }(78.1 \%) ; \\
\text { América Latina } \\
(12.5 \%)\end{array}$ \\
\hline $\begin{array}{l}\text { Kentucky Folklore } \\
\text { Record }\end{array}$ & $\begin{array}{l}\text { Kentucky Folklore } \\
\text { Society (EUA) }\end{array}$ & Inglés & & $0.1 \%$ & $0.2 \%$ & $\operatorname{EUA}(94.4 \%)$ \\
\hline Mélusine & $\begin{array}{l}\text { Editions d'Homme } \\
\text { (Francia) }\end{array}$ & Francés & & $0.9 \%$ & $2.8 \%$ & $\begin{array}{l}\text { Francia(66.0\%); } \\
\text { EUA (12.0\%) }\end{array}$ \\
\hline Millî Folklor & $\begin{array}{l}\text { Millî Folklor Dergisi } \\
\text { (Turquía) }\end{array}$ & Turco & $\begin{array}{l}\text { Inglés } \\
\text { Francés }\end{array}$ & $1.4 \%$ & $4.1 \%$ & Turquía (97.0\%) \\
\hline Mythlore & $\begin{array}{l}\text { Mythopoeic Society } \\
\text { (EUA) }\end{array}$ & Inglés & & $2.4 \%$ & $2.3 \%$ & EUA (85.4\%) \\
\hline New York Folklore & $\begin{array}{l}\text { New York Folklore } \\
\text { Society (EUA) }\end{array}$ & Inglés & & $1.3 \%$ & $2.8 \%$ & $\operatorname{EUA}(93.7 \%)$ \\
\hline $\begin{array}{l}\text { Österreichische } \\
\text { Zeitschrift für } \\
\text { Volkskunde }\end{array}$ & $\begin{array}{l}\text { Österreichische } \\
\text { Zeitschrift für } \\
\text { Volkskunde } \\
\text { Museo de folclore } \\
\text { y arte popular de } \\
\text { Austria }\end{array}$ & Alemán & $\begin{array}{l}\text { Inglés } \\
\text { Francés }\end{array}$ & $7.2 \%$ & $5.9 \%$ & $\begin{array}{l}\text { Austria }(66.9 \%) \text {; } \\
\text { Alemania }(18.3 \%)\end{array}$ \\
\hline $\begin{array}{l}\text { Revista de Dialecto- } \\
\text { logía y Tradiciones } \\
\text { Populares }\end{array}$ & $\begin{array}{l}\text { Consejo Superior } \\
\text { de Investigaciones } \\
\text { Científicas } \\
\text { (España) }\end{array}$ & Español & $\begin{array}{l}\text { Inglés } \\
\text { Francés } \\
\text { Portugués } \\
\text { Alemán }\end{array}$ & $2.9 \%$ & $6.0 \%$ & $\begin{array}{l}\text { España }(77.7 \%) ; \\
\text { América Latina } \\
(8.9 \%) ; \\
\text { EUA }(5.8 \%)\end{array}$ \\
\hline $\begin{array}{l}\text { Revista de Etnografie } \\
\text { si Folclor }\end{array}$ & $\begin{array}{l}\text { Academia Românâ } \\
\text { (Academia de } \\
\text { Rumania) }\end{array}$ & Inglés & $\begin{array}{l}\text { Francés } \\
\text { Alemán } \\
\text { Italiano }\end{array}$ & $0.2 \%$ & $0.5 \%$ & $\begin{array}{l}\text { Rumanía (42.9\%); } \\
\text { Hungría (16.3\%); } \\
\text { Alemania (8.2\%); } \\
\text { Bulgaria (6.1\%); } \\
\text { RU (6.1\%) }\end{array}$ \\
\hline $\begin{array}{l}\text { Schweizerisches } \\
\text { Archiv für Volkskunde }\end{array}$ & $\begin{array}{l}\text { Schweizerisches Ar- } \\
\text { chiv für Volkskunde } \\
\text { (Archivo de folclore } \\
\text { de Suiza) }\end{array}$ & Alemán & $\begin{array}{c}\text { Francés } \\
\text { Italiano } \\
\text { Inglés }\end{array}$ & $6.8 \%$ & $4.1 \%$ & $\begin{array}{l}\text { Suiza (53.8 \%); } \\
\text { Alemania (29.0\%); } \\
\text { Austria (5.9\%) }\end{array}$ \\
\hline $\begin{array}{l}\text { Southern Folklore } \\
\text { Quarterly }\end{array}$ & $\begin{array}{l}\text { South Atlantic } \\
\text { Modern Language } \\
\text { Association \& } \\
\text { University of Florida } \\
\text { (EUA) }\end{array}$ & Inglés & & $0.3 \%$ & $0.5 \%$ & $\operatorname{EUA}(91.2 \%)$ \\
\hline
\end{tabular}




\begin{tabular}{|c|c|c|c|c|c|c|}
\hline Ulster Folklife & $\begin{array}{l}\text { Ulster Folk and } \\
\text { Transport Museum } \\
\text { (Uster, RU) }\end{array}$ & Inglés & & $1.5 \%$ & $2.4 \%$ & $\begin{array}{l}\operatorname{RU}(79.8 \%) ; \\
\text { Irlanda }(12.8 \%) ; \\
\text { EUA }(5.3 \%)\end{array}$ \\
\hline $\begin{array}{l}\text { Voices. The Journal } \\
\text { of New York Folklore }\end{array}$ & $\begin{array}{l}\text { New York Folklore } \\
\text { Society (EUA) }\end{array}$ & & & $1.4 \%$ & $2.2 \%$ & $\operatorname{EUA}(97.1 \%)$ \\
\hline Volkskunde & $\begin{array}{l}\text { Centrum voor Studie } \\
\text { en Documentatie } \\
\text { (Centro para la } \\
\text { Investigación y la } \\
\text { Documentación, } \\
\text { Bélgica) }\end{array}$ & Holandés & $\begin{array}{l}\text { Alemán } \\
\text { Inglés } \\
\text { Francés }\end{array}$ & $4.1 \%$ & $5.4 \%$ & $\begin{array}{l}\text { Bélgica (87.4\%); } \\
\text { Holanda (10.6\%) }\end{array}$ \\
\hline Western Folklore & $\begin{array}{l}\text { Western States } \\
\text { Folklore Society } \\
\text { (EUA) }\end{array}$ & Inglés & & $5.4 \%$ & $5.9 \%$ & $\operatorname{EUA}(92.1 \%)$ \\
\hline $\begin{array}{l}\text { Zeitschrift für } \\
\text { Volkskunde }\end{array}$ & $\begin{array}{l}\text { Deutsche Gesells- } \\
\text { chaft für Volkskunde } \\
\text { (Sociedad de Folclo- } \\
\text { re de Alemania) }\end{array}$ & Alemán & Inglés & $10.5 \%$ & $4.5 \%$ & $\begin{array}{l}\text { Alemán }(77.0 \%) ; \\
\text { Austria }(7.1 \%) ; \\
\text { Suiza }(6.1 \%)\end{array}$ \\
\hline
\end{tabular}

Si nos centramos exclusivamente en los documentos considerados artículos (articles) por WoS, el número de trabajos se eleva a 9726 . El 16.2 \% de estos registros también ha sido incluido en otra categoría temática además del folclore y el $1.6 \%$ a tres o más categorías. La categoría predominante es la literatura, que apareció en 476 registros, seguida de Estudios de Asia (455), Humanidades-Multidiciplinar (283), Música (261), Arqueología (152), Antropología (152) y finalmente Historia (104). Pero es necesario recordar que esta categoría de WoS se asigna a los títulos de revista y no a cada artículo de manera específica.

El $58.9 \%$ de los artículos son en inglés; $19.6 \%$ en alemán; $8 \%$ en español; $5.3 \%$ en holandés (o flamenco); $4.1 \%$ en turco; $3.9 \%$ en francés y el restante $0.3 \%$, sólo 27 documentos, en portugués, italiano, ruso y griego, y dos que son multilingües. En el caso de reseñas de libros, la lengua dominante es el alemán en el $51.4 \%$ de los casos, seguido de inglés en el $42.1 \%$ y, muy por detrás, holandés (2.7 \%) y español (2.3\%). Sin embargo, el $92 \%$ de los documentos en actas (proceedings) son en inglés. La evolución cronológica de la presencia en WoS de referencias a documentos escritos en lenguas diferentes al inglés depende mucho de la adición de títulos de revista a la base de datos o de su desaparición. Por ejemplo, la aparición de artículos en turco se produce sólo con la indexación de la revista Millî Folklor en 2007.

\section{Productividad, colaboración y distribución geográfica}

El número total de autores diferentes de artículos asciende a 6 176. En 64 referencias no aparece el nombre del investigador. La Tabla 3 muestra los 15 
autores que han escrito 20 artículos o más. Son reconocidos folcloristas y etnólogos que han utilizado principalmente la lengua de su país de nacimiento o de adopción profesional. Cabe señalar que tres de ellos sólo han publicado en una revista indexada en WoS. La situación se vería modificada si consideráramos las reseñas. Por ejemplo, el folclorista suizo Robert Widhaber (19021982) es el autor con más documentos indexados. De los 336 registros, 332 son reseñas de libros, uno es una autobiografía y sólo tres son identificados realmente como artículos por WoS.

Tabla 3. Autores con más artículos en WoS

\begin{tabular}{|c|c|c|c|c|}
\hline Autor & País (Afiliación) & $\begin{array}{l}\text { Idioma usado } \\
\text { principalmente } \\
\text { (con \% del total) }\end{array}$ & $\begin{array}{l}\mathrm{N}^{0} \text { total } \\
\text { artículos }\end{array}$ & $\begin{array}{l}\mathrm{N}^{0} \text { total revistas } \\
\text { diferentes de los } \\
\text { artículos }\end{array}$ \\
\hline $\begin{array}{l}\text { Braekman, Willy } \\
\text { Louis }\end{array}$ & Bélgica (Flandes) & Holandés (97.4 \%) & 78 & 1 \\
\hline Dundes, Alan & EUA & Inglés (97.2 \%) & 36 & 8 \\
\hline Top, Stefaan & Bélgica (Flandes) & Holandés (94.3 \%) & 35 & 3 \\
\hline Schindler, Margot & Austria & Alemán (100 \%) & 32 & 1 \\
\hline Smith, John B & Reino Unido (Inglaterra) & Inglés (100 \%) & 29 & 6 \\
\hline Bendix, Regina & $\begin{array}{c}\text { Alemania } \\
\text { (nacida en Suiza) }\end{array}$ & Inglés (64 \%) & 25 & 7 \\
\hline Abrahams, Roger D, & EUA & Inglés (100 \%) & 24 & 6 \\
\hline Thijs, Alfons K, L, & Bélgica (Flandes) & Holandés (95.7 \%) & 23 & 1 \\
\hline Tschofen, Bernhard & $\begin{array}{l}\text { Suiza (actualmente) } \\
\text { Alemania, Austria } \\
\text { (anteriormente) } \\
\text { (nacida en Alemania) }\end{array}$ & Alemán (100%) & 23 & 3 \\
\hline Bausinger, Hermann & Alemania & Alemán (72.7%) & 22 & 6 \\
\hline Bronner, Simon J, & EUA (nacido en Israel) & Inglés (100 \%) & 21 & 7 \\
\hline Köstlin, Konrad & $\begin{array}{c}\text { Austria } \\
\text { Alemania (anteriormente) } \\
\text { (nacido en Alemania) }\end{array}$ & Alemán (90.4%) & 21 & 5 \\
\hline Lindahl, Carl & EUA & Inglés (100 \%) & 21 & 5 \\
\hline Oring, Elliott & EUA & Inglés (100 \%) & 21 & 6 \\
\hline Mieder, Wolfgang & $\begin{array}{c}\text { EUA } \\
\text { (nacido en Alemania) }\end{array}$ & Inglés (85.0 \%) & 20 & 11 \\
\hline $\begin{array}{l}75 \text { autores entre } 10 \\
\text { y } 19 \text { artículos }\end{array}$ & & & 978 & \\
\hline
\end{tabular}




\begin{tabular}{|l|l|l|c|c|}
\hline $\begin{array}{l}378 \text { autores entre } 4 \\
\text { y } 9 \text { artículos }\end{array}$ & & 1991 & \\
\hline $\begin{array}{l}306 \text { autores } \\
\text { con } 3 \text { artículos }\end{array}$ & & & 918 & \\
\hline $\begin{array}{l}784 \text { autores } \\
2 \text { artículos }\end{array}$ & & 1568 & \\
\hline $\begin{array}{l}4618 \text { autores } \\
\text { con } 1 \text { artículo }\end{array}$ & & & 4618 & \\
\hline
\end{tabular}

La distribución del número de autores en relación a la producción sigue una función potencial que se ajusta a la expresión numérica de la ley de Lotka QUOTE con una desviación máxima absoluta de 0.006 en la prueba de Kolmogorov-Smirnov.

El porcentaje de artículos con un solo autor es de $93.4 \%$; del $6.6 \%$ restante con coautoría, $5.3 \%$ los firman dos autores; $0.9 \%$ tres y $0.4 \%$ de cuatro o más, con un valor máximo de 17 . Naturalmente el valor de la mediana es exactamente 1 y el de la media es de 1.09. Los resultados son similares en el caso de las actas de congresos (proceedings) donde el $9.17 \%$ pertenece a un solo autor. En las reseñas de libros y otros tipos de documentos la coautoría es aún menos frecuente. Por otra parte, no se han observado cambios en esta tendencia en los últimos años.

Estos resultados coinciden estrechamente con el estudio de Yalçin (2010) de la revista turca Millî Folklor correspondiente al periodo 2007-2009. Dicho autor encontró que de un total de 180 artículos, 97 \% tenían un solo autor; cuatro artículos con dos autores; un artículo con tres autores y otro artículo con un número mayor de firmas. Además, afirma que más del $98 \%$ de los artículos de Millî Folklor fueron escritos por autores turcos. En el presente estudio los resultados obtenidos muestran que EUA contribuye con un $37.3 \%$, seguido por el Reino Unido (9.5\%), Alemania (9.4 \%), España (5.2 \%), Turquía (5.2\%) y Austria ( $5.1 \%$ ), hasta un total de 97 países.

\section{Artículos, autores y revistas más citados}

El artículo más citado es "Tradition, genuine or spurious" de Richard Handler y Jocelyn Linnekin, publicado en el American Journal of Folklore, con 253 citas. De hecho, 24 de los 25 artículos más citados son de esta publicación. El primer artículo de otra revista (Folklore) no se encuentra hasta la 19a. posición. La Tabla 4 muestra los 12 artículos con más de 50 citas. Todos estos autores son de EUA por nacimiento o adopción profesional. 
Entre las ponencias (proceedings), el documento más citado es "The Symbolic Ur-Meaning of Shamanism and Performing Arts" (Shamanism in Performing Arts Conference), del folclorista coreano Tae-Gon Kim. Este trabajo ha recibido 66 citas, mientras que el resto de las ponencias obtienen seis citas o menos. Entre el resto de tipos de documento el más citado es la editorial "Theorizing the Hybrid" de un número especial del Journal of American Folklore, firmado por los estadounidenses Deborah A. Kapchan y Pauline Turner Strong. Esta contribución obtiene 46 citas, y es seguida por dos reseñas de libros de la misma revista, con 31 y 29 citas respectivamente.

Tabla 4. Artículos con más de 50 citas

\begin{tabular}{|l|l|c|c|}
\hline \multicolumn{1}{|c|}{ Autor } & \multicolumn{1}{|c|}{ Título } & $\begin{array}{c}\text { Año de } \\
\text { publicación }\end{array}$ & N $^{\circ}$ citas \\
\hline $\begin{array}{l}\text { Handler, Richard; Linne- } \\
\text { kin, Jocelyn }\end{array}$ & Tradition, genuine or spurious & 1984 & 253 \\
\hline Ben-Amos, Dan & Toward a definition of folklore in context & 1971 & 114 \\
\hline Abrahams, Roger D. & Introductory remarks to a rhetorical theory of folklore & 1968 & 101 \\
\hline Georges, Robert A. & Toward an understanding of storytelling events & 1969 & 85 \\
\hline Dundes, Alan & From etic to emic units in the structural study of folktales & 1962 & 79 \\
\hline Abrahams, Roger D. & Phantoms of romantic nationalism in folkloristics & 1993 & 72 \\
\hline Wolf, Eric Robert & The virgin of Guadalupe - a mexican national symbol & 1958 & 68 \\
\hline Bascom, William R. & The forms of folklore - prose narratives & 1965 & 66 \\
\hline Hymes, Dell Hathaway & Folklores nature and suns myth & 1975 & 62 \\
\hline Robinson, John A. & Personal narratives reconsidered & 1981 & 62 \\
\hline Abrahams, Roger D. & Playing the dozens & 1962 & 61 \\
\hline Kalcik, Susan & $\begin{array}{l}\text { "...Like Ann's gynecologist or time. I was almost raped". } \\
\text { Personal narratives in Women's Rap Groups }\end{array}$ & 1975 & 53 \\
\hline
\end{tabular}

En total, el conjunto de documentos reciben un total de 17609 citas. El autor más citado es Alan Dundes (1934-2005), folclorista de la University of California, Berkeley (Tabla 5). También obtiene el índice $b$ más alto, con un valor de 11. En un sentido muy general, y como cabría esperar, cuanto mayor es el número de citas mayor es el índice $h$. Sin embargo, hay una excepción notable: los antropólogos culturales estadounidenses Richard Handler y Jocelyn Linnekin obtienen la mayoría de sus citas de un solo artículo. Su contribución es citada desde varias disciplinas, debido a su profundización del concepto de tradición y su relación con el nacionalismo. A pesar de que los autores más citados obtienen la mayoría de las citas por sus artículos, hay 
excepciones. Por ejemplo, la profesora estadounidense Deborah Kapchan sólo alcanza el $26.5 \%$ con este tipo de documento.

Tabla 5. Autores con al menos 100 citas

\begin{tabular}{|c|c|c|c|c|c|}
\hline Autor & $\begin{array}{l}\mathrm{N}^{0} \text { total } \\
\text { de citas }\end{array}$ & Índice $\mathrm{h}$ & $\begin{array}{l}\mathrm{N}^{0} \text { total de } \\
\text { documentos } \\
\text { citados }\end{array}$ & $\begin{array}{l}\mathrm{N}^{0} \text { de citas } \\
\text { en artículos }\end{array}$ & $\begin{array}{l}\% \text { de citas } \\
\text { de artículos } \\
\text { respecto del } \\
\text { total de citas } \\
\text { recibidas }\end{array}$ \\
\hline Dundes, Alan & 414 & 11 & 38 & 402 & $97.1 \%$ \\
\hline Abrahams, Roger D. & 343 & 8 & 29 & 330 & $96.2 \%$ \\
\hline Handler, Richard & 259 & 2 & 2 & 253 & $97.7 \%$ \\
\hline Linnekin, Jocelyn & 253 & 1 & 1 & 253 & $100.0 \%$ \\
\hline Georges, Robert A. & 232 & 6 & 20 & 221 & $95.3 \%$ \\
\hline Ben-Amos, Dan & 217 & 6 & 16 & 184 & $84.8 \%$ \\
\hline Oring, Elliott & 179 & 8 & 27 & 140 & $78.2 \%$ \\
\hline Kirshenblatt-Gimblett, B. & 146 & 6 & 9 & 85 & $58.2 \%$ \\
\hline Bauman, Richard & 145 & 6 & 12 & 123 & $84.8 \%$ \\
\hline Fine, Gary Alan & 144 & 7 & 19 & 126 & $87.5 \%$ \\
\hline Degh, Linda & 130 & 7 & 14 & 99 & $76.2 \%$ \\
\hline Bendix, Regina & 110 & 6 & 21 & 104 & $94.5 \%$ \\
\hline Hymes, Dell Hathaway & 108 & 4 & 6 & 106 & $98.1 \%$ \\
\hline Bascom, William R. & 104 & 5 & 6 & 92 & $88.5 \%$ \\
\hline Kapchan, Deborah & 102 & 4 & 4 & 27 & $26.5 \%$ \\
\hline
\end{tabular}

Con referencia a las revistas más citadas, el primer lugar lo ocupa el Journal of American Folklore (JAF), que obtiene el $45.1 \%$ de las citas y una media de 1.53 citas por artículo. Su índice $b$ alcanza el valor 30. JAF es seguida a gran distancia por Western Folklore, con un 10.7 \% de las citas y un índice $b$ 15. A continuación vienen Journal of the Folklore Institute / Journal of Folklore Research (8.0 \%) y Folklore (6.9 \%), entre las revistas con más de 200 citas (Tabla 6). La mediana es de 156 citas y la media es de 600 citas por título. El estudio de Yalçin (2010) en Millî Folklor también obtuvo que era la revista más citada (22.7\%), seguida de Zeitschrift für Volkskunde (13.5\%). 
Tabla 6. Revistas que reciben más de 200 citas

\begin{tabular}{|c|c|c|c|c|c|c|}
\hline Título de la revista & $\begin{array}{c}\mathrm{N}^{\circ} \text { de } \\
\text { Contribuciones }\end{array}$ & $\begin{array}{l}\text { Total de } \\
\text { citas }\end{array}$ & $\begin{array}{c}\% \\
\text { de citas }\end{array}$ & $\begin{array}{c}\% \\
\text { acumulado } \\
\text { de citas }\end{array}$ & $\begin{array}{c}\text { Citas por } \\
\text { contribución }\end{array}$ & Índice $\mathrm{h}$ \\
\hline Journal of American Folklore & 4800 & 7843 & $45.1 \%$ & $45.1 \%$ & 1.63 & 30 \\
\hline Western Folklore & 1853 & 1865 & $10.7 \%$ & $55.8 \%$ & 1.01 & 15 \\
\hline $\begin{array}{l}\text { Journal of the Folklore Institute. } \\
\text { Más tarde: Journal of Folklore } \\
\text { Research }\end{array}$ & 694 & 1399 & $8.0 \%$ & $63.9 \%$ & 2.02 & 13 \\
\hline Folklore & 1867 & 1195 & $6.9 \%$ & $70.7 \%$ & 0.64 & 9 \\
\hline Fabula & 3069 & 895 & $5.1 \%$ & $75.9 \%$ & 0.29 & 9 \\
\hline $\begin{array}{l}\text { Asian Ethnology, } \\
\text { Más tarde: Asian Folklore } \\
\text { Studies }\end{array}$ & 1611 & 678 & $3.9 \%$ & $79.8 \%$ & 0.42 & 7 \\
\hline Zeitschrift für Volkskunde & 3422 & 674 & $3.9 \%$ & $83.6 \%$ & 0.20 & 9 \\
\hline Journal of Latin American Lore & 91 & 380 & $2.2 \%$ & $85.8 \%$ & 4.18 & 9 \\
\hline Folk Music Journal & 1176 & 305 & $1.8 \%$ & $87.6 \%$ & 0.26 & 7 \\
\hline New York Folklore & 538 & 296 & $1.7 \%$ & $89.3 \%$ & 0.55 & 7 \\
\hline Volkskunde & 1360 & 230 & $1.3 \%$ & $90.6 \%$ & 0.17 & 4 \\
\hline $\begin{array}{l}\text { Schweizerisches Archiv für } \\
\text { Volkskunde }\end{array}$ & 2211 & 227 & $1.3 \%$ & $91.9 \%$ & 0.10 & 6 \\
\hline $\begin{array}{l}\text { Revista de Dialectología y } \\
\text { Tradiciones Populares }\end{array}$ & 987 & 212 & $1.2 \%$ & $93.1 \%$ & 0.21 & 5 \\
\hline Ulster Folklife & 487 & 206 & $1.2 \%$ & $94.3 \%$ & 0.42 & 5 \\
\hline
\end{tabular}

Para estimar un valor para los factores de impacto se ha utilizado el Scimago Journal Rank-SJR, basado en los datos de Scopus, puesto que el A\&HCI no cuenta hasta la fecha de JCR. Puesto que el SJR no dispone de una categoría que identifique las revistas de folclore, se han seleccionado manualmente. La categoría temática de Estudios Culturales del SJR agrupa más de 450 revistas. JAF se clasifica dentro de esta categoría. Sin embargo, Folklore, una publicación equivalente británica, se clasifica en la categoría de Historia. Western Folklore se encuentra en dos categorías: en Antropología y Artes Visuales y Escénicas.

La Figura 2 muestra la evolución del factor de impacto (FI) de las seis revistas con un valor medio superior en el SJR entre 2003 y 2012. El valor mayor de FI fue alcanzado por JAF en 2010 (0.270), con una media para el periodo de 0.155. 


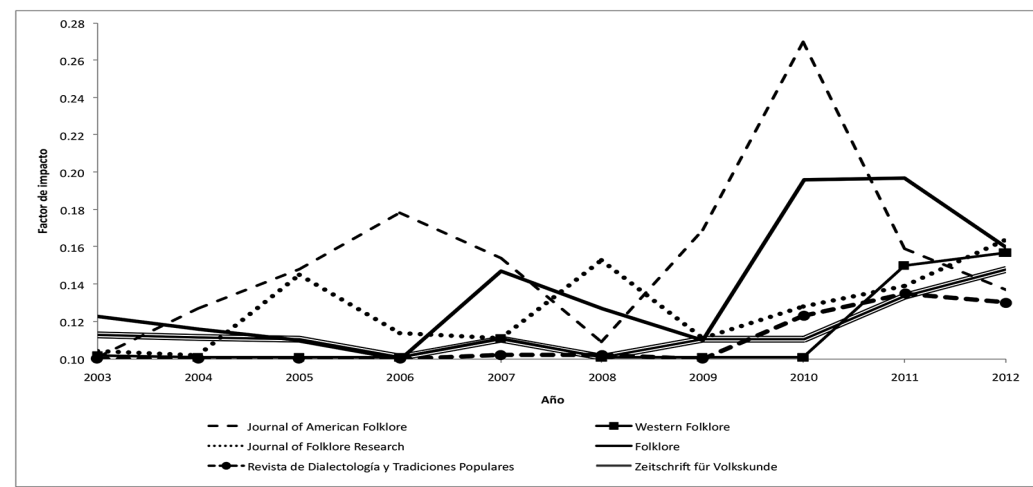

\section{Obsolescencia}

Con referencia a la obsolescencia sincrónica, la vida media es de 16 años, incluyendo tanto fuentes primarias como secundarias, siendo los documentos más antiguos del año 1500 (Collectanea Adagiorum y Den Herbarius in Dyetsche). El índice de Price correspondiente al porcentaje de referencias de menos de cinco años es de un $15.9 \%$, resultando la media aritmética de 30.7 años. La obsolescencia se reduce a nueve años si solamente se consideran las citas a los artículos sobre folclore indexados en WoS, los cuales naturalmente son fuentes secundarias. Esta diferencia en la obsolescencia no debería atribuirse exclusivamente a la presencia de fuentes primarias en el primer caso, puesto que diversos estudios han mostrado una obsolescencia más lenta de las contribuciones publicadas en las monografías frente a la que se produce con los artículos de revista (Wolfe Thompson, 2002; Ardanuy, Urbano y Quintana, 2009). Para las citas en los artículos de folclore de WoS el índice de Price alcanza un $33.2 \%$ y la antigüedad media decae hasta los 12.2 años.

No se dispone de muchos datos para comparar específicamente dentro del área del folclore. Yalçin (2010) estimó en un valor de 11.8 años la mediana de la antigüedad de las referencias citadas en Millî Folklor entre 2007 y 2009. Ardanuy (2013b) obtuvo en su análisis de la revista catalana de folclore L'Upir un valor de 20 años si se consideran todas las citas, pero se reducía a 14 si se contabilizaban únicamente las fuentes secundarias. En este último artículo el índice de Price para las fuentes secundarias fue de $31.8 \%$. 


\section{Conclusiones}

Este trabajo muestra la posibilidad de acercarse al conocimiento de una disciplina minoritaria y escasamente estudiada de las humanidades, como es el folclore, desde una perspectiva bibliométrica. Este análisis es posible gracias a una herramienta de búsqueda introducida en WoS en 2012 y que había sido reclamada desde la investigación bibliométrica con anterioridad (Ardanuy, Urbano y Quintana, 2011). En versiones anteriores esta opción solamente existía como una herramienta de filtrado sobre los resultados (filtrado expost). Igualmente sería de gran utilidad la aparición o desglose de otras categorías en WoS y en Scopus. En este último caso no existe la mencionada categoría folclore y revistas destacadas del área como JAF, Western Folklore y Folklore se encuentran bajo clasificaciones diferentes en el SJR.

Al observar los resultados obtenidos en el presente análisis, aparece un claro predominio de revistas estadounidenses. Algo más de un tercio son de este país y un poco más de la mitad publican sólo artículos en inglés. Pero el bajo nivel de citas recibidas en varios casos, así como el hecho de que tienen una orientación regional en su contenido, justificarían la inclusión de otras revistas de folclore en WoS, cuyos perfiles de citas recibidas y política de publicación no deberían alejarse mucho de algunas de las presentes. Ello facilitaría análisis de citas detallados.

El presente estudio indica también la gran importancia de reseñas de libros, que en conjunto representan más de la mitad de los registros, no limitándose a los trabajos de corte académico, mostrando así que los investigadores del área estiman que puede ser conveniente estar informado sobre fuentes más divulgativas y otras de corte paranormal, quizás para conocer las limitaciones de tales fuentes, pero también como materia de ulterior estudio debido a que el folclore, como expresión cultural, se encuentra en perpetuo cambio.

Por otra parte, aunque no se han aportado datos estadísticos, se ha constatado la importancia en el área de las monografías y los libros en general, lo que debería considerarse para una evaluación global de la disciplina y de sus agentes. Junto a esta característica aparecen otras también típicas de las disciplinas de humanidades (Stone, 1982; Watson-Boone, 1994), como que la colaboración sigue siendo inusual o la orientación regional y local de muchos trabajos, lo que suele traducirse en el hecho de que los autores tienden a publicar profusamente en otros idiomas diferentes al inglés. Los valores de obsolescencia son también los habituales en humanidades. En resumen, los resultados obtenidos confirman los patrones tradicionales de comunicación de las áreas de humanidades. Desde esta perspectiva, no parece muy útil disponer por el momento de un JCR en el área. 
Finalmente, los datos específicos obtenidos sobre la disciplina permiten identificar nítidamente aspectos relevantes en el campo del folclore, como el hecho de que la revista más influyente sea American Journal of Folklore o el papel muy destacado del investigador ya fallecido Alan Dundes.

\section{REFERENCIAS}

Aghaei Chadegani, Arezoo, Hadi Salehi, Melor Md Yunus, Hadi Farhadi, Masood Fooladi, Maryam Farhadi y Nader Ale Ebrahim. 2013. "A Comparison between Two Main Academic Literature Collections: Web of Science and Scopus Databases". Asian Social Science 9 (5): 18-26.

Ardanuy, Jordi, Cristóbal Urbano y Lluís Quintana. 2009. "A citation analysis of Catalan literary studies (1974-2003): Towards a bibliometrics of humanities studies in minority languages". Scientometrics 81 (2):347-366.

Ardanuy, Jordi, Cristóbal Urbano y Lluís Quintana. 2011. "Use of the A\&HCI to evaluate research in minority language literatures: the case of Catalan and Danish literature". BiD: textos universitaris de biblioteconomia i documentació 27. Fecha de consulta: 25 de enero de 2015. http://bid.ub.edu/27/ardanuy3.htm

Ardanuy, Jordi. 2013a. "Sixty Years of Citation Analysis Studies in the Humanities (1951-2010)". Journal of the American Society for Information Science and Techno$\log 64$ (8): 1751-1755.

Ardanuy, Jordi. 2013b. "L'impacte de l'ús de fonts documentals primàries en l'anàlisi bibliomètrica en humanitats: el cas del folklore”. L'Upir 35: 13-22. Fecha de consulta: 25 de enero de 2015. http://www.cercle-v.org/Pdf/upir35_3p.pdf

Calvo, Luis. 2002. "La Revista de Dialectología y Tradiciones Populares y sus aportaciones a la antropología española (1944-1996)". Revista de Dialectología y Tradiciones Populares 57 (1): 29-58.

Chang, Cuiyin. 2006. "Bibliometric Analysis of Folklore Studies". Folklore Studies 1: 242-250.

Dundes, Allan. 2005. "Folkloristics in the Twenty-First Century (AFS Invited Presidential Plenary Address, 2004)”. Journal of American Folklore 118: 385-408.

Engels, Tim C. E., Truyken L. B. Ossenblok y Eric H. J. Spruyt. 2012. "Changing publication patterns in the Social Sciences and Humanities, 2000-2009”. Scientometrics 93 (2):373-390.

Hicks, Diana. 2004. "Comprehensive statistics", en Handbook of Quantitative Science and Technology Research: The Use of Publication and Patent Statistics in Studies of SET Systems, H. F. Moed, W. Glänzel y U. Schmoch (eds.), 473-496. New York: Springer.

Wolfe Thompson, Jennifer. 2002. "The Death of the Scholarly Monograph in the Humanities? Citation Patterns in Literay Scholarship”. Libri 52 (3): 121-136.

Leydesdorff, Loet e Ismael Rafols. 2009. "A global map of science based on the ISI subject categories". Journal of the American Society for Information Science and Technology 60 (2): 348-362. 
Leydesdorff, Loet, Stephen Carley e Ismael Rafols. 2013. "Global maps of science based on the new Web-of-Science categories”. Scientometrics 94 (2): 589-593.

Moed, Henk F. 2005. Citation Analysis in Research Evaluation. Dordrecht: Springer.

Nederhof, Anton J. 2006. "Bibliometric monitoring of research performance in the Social Sciences and the Humanities: a review”. Scientometrics 66 (1): 81-100.

Osca-Lluch, Julia, Ana Veyrat y Jesús Morales. 2013. "El consumo de información en Humanidades”. Arbor 189: a026. Fecha de consulta: 25 de enero de 2015. http:// arbor.revistas.csic.es/index.php/arbor/article/view/1567/1630

Rudy, Jill Terry. 2002. "Toward an Assessment of Verbal Art as Performance: A Cross-Disciplinary Citation Study with Rhetorical Analysis". Journal of American Folklore 115: 5-27.

Stone, Sue. 1982. "Progress in documentation. Humanities scholars: Information needs and uses". Journal of Documentation 38 (4): 292-313.

Thukakwenzeka, Clifford Dube. 2003. "Bibliometric Analysis of indigenous Knowledge in SABINET Databases", en Towards Popularizing Library and Information Science (LIS) Research: Proceedings of the 4th annual DLIS/LISA Conference, D. N. Ocholla y L. Dube (eds.), 38-44. Kwadlangezwa: Department of Library and Information Science, University of Zululand. Fecha de consulta: 25 de enero de 2015. http://citeseerx.ist.psu.edu/viewdoc/download?doi=10.1.1.129.5386\&re$\mathrm{p}=$ rep1\&type $=\mathrm{pdf}$

Van Impe, Steven y Ronald Rousseau. 2006. "Web-to-print citations and the humanities”. Information. Wissenschaft und Praxis 57 (8): 422-426.

Watson-Boone, R. 1994. "The Information Needs and Habits of Humanities Scholars”. Reference Quaterly 34 (2): 203-215.

Widdowson, John David Allison. 2010. "Folklore Studies in English Higher Education: Lost Cause or New Opportunity?”. Folklore 121 (2): 125-142.

Yalçin, Haydar. 2010. "Millî Folklor dergisinin bibliyometrik profili (2007-2009)". Millî Folklor 85: 205-211.

Zheng, Le-dan. 2009. "Bibliometrical Analysis of China' s Intangible Cultural Heritage Study during the Last Five Years”. Guizhou Ethnic Studies 31 (2):39-44.

Zuccala, Alesia y Thed van Leeuwen. 2011. "Book reviews in humanities research evaluations". Journal of the American Society for Information Science and Techno$\operatorname{logy} 62$ (10): 1979-1991.

Para citar este texto:

Ardany, Jordi. 2017. "El uso de Web of Science para el estudio de disciplinas minoritarias en Humanidades': el caso del folclore". Investigación Bibliotecológica: archivonomia, bibliotecología e información 73 (31): 111-129.

http://dx.doi.org/10.22201/iibi.24488321xe.2017.73.57849 